\title{
Ongoing strategies against mitochondrial diseases: prevention and development of genetic therapies
}

\author{
Combatiendo las enfermedades mitocondriales: estrategias para el desarrollo \\ de terapias génicas y estrategias de prevención
}

\author{
Diego González Halphen*
}

Mitochondria are organelles present in the cytosol of most eukaryotic organisms. They represent the cell's main source of metabolic energy since they generate ATP (adenosine-triphosphate) through oxidative phosphorylation, a process involving the respiratory chain and the ATP synthase. Mitochondria also participate in other metabolic processes such as the synthesis of several cofactors and fatty acids, and have a role in metabolism regulation, cellular cycle control, ageing, and programmed cell death. These organelles originated approximately 2,500 million years ago, when an alpha-proteobacterium was engulfed by another cell, probably an archaeon (archaebacterium) and a symbiotic relationship was thus established between the two micro-organisms. With time, intra-cellular alpha-proteobacteria gradually transferred their genetic material to the host's nucleus, eventually giving rise to new organelles in the eukaryotic cell. For the aforementioned reasons, mitochondria preserve several remnants of their bacterial origin, among them, the presence of a small genome, which in humans is a circular molecule of 16,600 base pairs, which we will herein refer to as mitochondrial DNA (mtDNA). Information contained within this genetic material represents less than $0.2 \%$ of the cells' total genetic information; the remaining $99.8 \%$ is found in the nuclear chromosomes (around 19,000 genes which encode proteins). The human mtDNA contains 37 genes which encode ribosomal RNAs, transfer RNAs and 13 proteins that participate in oxidative phosphorylation. These set of highly hydrophobic membrane proteins are synthesized in mitochondrial ribosomes, integrated into the mitochondrial inner membrane, and assembled into the oxidative phosphorylation complexes. The rest of the 1,500 to 2,000 proteins that are found in mitochondria are encoded in the nuclear genome, are synthesized in the cytosol, and penetrate the mitochondria through a transport apparatus built by protein receptors and transporters located in the mitochondrial membranes. Thus, mitochondria imports over $98 \%$ of all proteins required for its function, while it synthesizes for itself only a dozen proteins, which have an essential role in oxidative phosphorylation. In this scenario, I like to compare mitochondria with an oil-producing country that imports all its supplies and exports energy in exchange (oil or ATP).

Like any genetic material, mtDNA is prone to suffer mutations, some of which can elicit a heterogeneous spectrum of human diseases, called mitochondrial syndromes or mitochondrial encephalomyopathies. These pathological conditions mainly affect tissues and organs which have high energy demands, such as the brain, skeletal muscle and the endocrine system. The following are some of the clinical manifestations of mitochondrial diseases: muscle weakness, intolerance to exercise, blindness, mental retardation, dementia, progressive epilepsy, sensory neuropathies, ataxia and renal dysfunction. Mitochondrial alterations have also been related to other metabolic defects such as diabetes, obesity, cardiovascular diseases, neurodegenerative syndromes and cancer. Mitochondrial diseases are not restricted to the presence of mutations in mtDNA, they can also be originated by mutations in the nuclear DNA, especially those which affect either the structure or function of proteins which will eventually internalize into mitochondria. Herein we will only focus on mtDNA defects.

mtDNA is maternally inherited. Thus, we humans possess the same mtDNA as our mothers, our grandmothers, our great-grandmothers. This is due to the fact that mitochondria are present only in the * Cellular Physiology Institute. National Autonomous University of
Mexico (UNAM).

This article can be read in its full version in the following page: http://www.medigraphic.com/facultadodontologiaunam 
spermatozoid's neck and are selectively destroyed once they reach the ovum, therefore, in general, there is no genetic contribution of paternal mtDNA during fertilization. Thus, several familial lineages that carry mitochondrial alterations exhibiting a clear maternal heredity pattern have been described.

To date, over 500 point mutations in mitochondrial genes related to human diseases have been reported, as well as several ablations and mtDNA duplications. Epidemiological studies have shown that approximately 1 out of 6,500 subjects may carry mtDNA pathogenic mutations. Mitochondria do not form anew in the cell; they can only be formed by the growth and division of pre-existing organelles. Even though there is a mitochondrial reticulum which is constantly fusing and dividing (experiencing fission), it is reckoned there are approximately $10 \mathrm{mtDNA}$ copies in each mitochondria, and there are between 100 and 1,000 mitochondria per cell (it is for this reason mtDNA is also useful in forensic sciences, since there are present more copies of mtDNA than of nuclear DNA, and therefore, is it possible to identify genetic material in very small samples, like in a hair follicle). For a mitochondrial disease to manifest itself, the point mutation in a mitochondrial gene must be present in at least $90 \%$ of the mtDNA population. Even though there are presently ongoing tools to transform nuclear DNA as well as various gene therapy protocols, to date there is no technique that allows the transformation of mtDNA of multi-cellular organisms, therefore, direct correction of human mtDNA mutations is not yet possible. Transformation of mtDNA in multi-cellular organisms continues to represent a challenge for both basic and clinical research. For the aforementioned reason, there is no effective treatment for mitochondrial diseases, except in those rare cases in which mtDNA mutation is solely found in one particular organ and there is a possibility of carrying out a transplant. Other therapeutic measures such as physical exercise and a variety of pharmacological therapies are only palliative measures, since they only mitigate some of the symptoms. The present lack of effective treatments for mitochondrial diseases has triggered exploration of several strategies to develop future gene therapies:

1. Selective inhibition of mutant mtDNA propagation. This approach attempts to introduce into mitochondria enzymes that are able to specifically recognize and cut mutant mtDNA. In this way, only the mtDNA lacking mutations is able to propagate.

2. Mitochondrial expression vectors. This technique attempts to introduce recombinant mIDNA into the organelle, either whole or in fragments. It must contain a wild gene copy whose expression is controlled by mitochondrial gene promoters. This is a merely theoretical option, since, as mentioned beforehand, it is nowadays impossible to transform mitochondria in living multi-cellular organisms.

3. Allotopic expression. Mitochondrial genes are relocated to and expressed from the nucleus. The cytosol-synthesized wild-type protein is then internalized into mitochondria taking advantage of natural apparatus of protein import.

Allotopic expression is considered one of the most viable options for genetic treatment of diseases caused by mtDNA mutations, since there are already established techniques to introduce genetic material into the cell nucleus (using a variety of adenoviral vectors), therefore, in principle, the resulting protein should reach all the affected mitochondria present in the cell. The gene intended for allotopic expression must be modified in such a way that it is efficiently expressed in the nucleus. It must be noted that in humans, the genetic code and codon usage of mitochondrial genes differ from the ones of nuclear genes. For example, the UAG codon that encodes tryptophan in accordance with the mitochondrial genetic code is interpreted as a stop codon in the nuclear genetic code. Therefore, mitochondrial genes must be re-designed in such a way that their corresponding messenger RNAs are correctly translated in the cytosolic ribosomes. After it is synthesized, the protein is directed to the mitochondria by a signal sequence located in its amino-terminus, which is called mitochondrial targeting signal. These signals are constituted by a polypeptide chain of 20 to 30 aminoacids that functions as a postal code, ensuring the dispatch of cytosol-synthesized proteins specifically to mitochondria and not to any other organelle in the cell. Targeting signals are recognized by the protein transporter complex located in the mitochondria's internal and external membranes. After crossing both mitochondrial membranes and reaching the mitochondrial matrix, the signal is removed by a specific protease, giving rise to the mature protein. An alternative strategy developed in recent years is to allow the ingress of messenger RNAs into mitochondria, which seem to be imported through the same protein transport apparatus. These messenger RNAs also have specific signals which direct them to the mitochondria. Once in the mitochondrial matrix, the messenger RNA can be translated by mitochondrial ribosomes and the corresponding protein is synthesized. Thus, proteins can directly integrate into the mitochondrial inner 
membrane and assemble into its corresponding oxidative phosphorylation complex.

As stated above, to date, there is no effective therapy for mitochondrial diseases. Nevertheless, in recent years, interesting prevention alternatives have emerged. The first one is simply a prenatal diagnosis, which would allow a couple to decide whether or not to have children. The second one is spindle transfer, a technology based on the exchange of genetic materials followed by in vitro fertilization techniques.

When there is clear evidence of the presence of a mitochondrial mutation associated with a mitochondrial disease in a female, the technology to avoid this mutation to be inherited is currently available. This technology is based on the pioneering work of the group of Masahito Tachibana ${ }^{1,2}$ and colleagues working in several research institutes in Oregon (USA). Studies were first conducted with primates and then successfully carried out with human cells (Tachibana et al, 2009, 2013). ${ }^{2}$ In essence, ovules are taken from a donor female (with healthy mitochondria, no mutations) as well as from the female carrying mtDNA mutations (the mother who wants to bear a healthy child). The achromatic spindle is removed (the set of micro-tubules which go from the chromosomes centromeres to the centrioles at the poles during meiosis) from the donor's ovule, that is to say all nuclear genetic material is eliminated from the ovule. In parallel, the achromatic spindle is also removed from the future mother's ovule afflicted with mitochondrial mutations, and it is transferred to the donor's ovule. In this fashion, «reconstructed» ovules, that contain the mother's nuclear genetic material (intact), as well as the donor's mitochondrial genetic material (also intact) are generated. The reconstructed ovule is fertilized in vitro, and embryogenesis is allowed to develop until the time when it can be safely implanted in the maternal uterus. A normal pregnancy will result, giving rise to a healthy baby who will have $49.9 \%$ of the father's chromosomal genetic material, $49.9 \%$ of the mother's chromosomal genetic material and only $0.2 \%$ of the donor's genetic material (corresponding to the mitochondrial DNA). Therefore, stating that the baby is the product of «three parents» is not strictly true, although it does contain a genetic mosaic of three subjects. On February $3^{\text {rd }} 2015$, the House of Commons of the United Kingdom allowed this technique to be used in the clinic, giving the opportunity to females with mitochondrial mutations to bear healthy offspring. Thus, United Kingdom has become the first country to allow in vitro fertilization techniques to make use of the genetic material of a «second mother» in order to repair mutated mitochondrial DNA. Undoubtedly, this is another example where prevention advances faster than cure. United Kingdom has set an example for other countries to follow, including ours. Expedite legislation offers citizens to take advantage of the technological and scientific advances at the earliest opportunity, and not after a long waiting period of maybe decades.

In spite of spectacular advances in mitochondrial disease prevention, allotopic expression is still an intellectual and experimental challenge which deserves further study. Successful allotopic expression will eventually set the bases for developing a genetic therapy which might give hope to patients suffering mitochondrial syndromes.

\section{REFERENCES}

1. Tachibana $\mathrm{M}$ et al. Mitochondrial gene replacement in primate offspring and embryonic stem cells. Nature. 2009; 461 (7262): 367-372.

2. Tachibana $M$ et al. Human embryonic stem cells derived by somatic cell nuclear transfer. Cell. 2009; 153 (6): 1228-1238.

Mailing address:

Diego González Halphen

E-mail: dhalphen@correo.ifc.unam.mx 